
\title{
Research S Surate \\ Mathematical modeling shows that ball-rolling dung beetles can use dances to avoid competition
}

\section{Zhanyuan Yin}

University of California Los Angeles

Leif Zinn-Brooks ( $\nabla$ Izinnbrooks@hmc.edu )

Harvey Mudd College https://orcid.org/0000-0001-5445-9915

\section{Research Article}

Keywords: Dung beetles, Competition, Random walk, Optimization

Posted Date: March 31st, 2021

DOI: https://doi.org/10.21203/rs.3.rs-246497/v1

License: (c) (1) This work is licensed under a Creative Commons Attribution 4.0 International License. Read Full License

Version of Record: A version of this preprint was published at Theoretical Ecology on November 6th, 2021. See the published version at https://doi.org/10.1007/s12080-021-00523-9. 


\title{
Mathematical modeling shows that ball-rolling dung beetles can use dances to avoid competition
}

\author{
Zhanyuan Yin • Leif Zinn-Björkman*
}

Received: date / Accepted: date

\begin{abstract}
Ball-rolling dung beetles shape a portion of dung into a ball and roll it away from the dung pile for later burial and consumption. These beetles perform dances (rotations and pauses) atop their dung balls in order to choose an initial rolling direction and to correct their rolling direction (reorient). Previous mathematical modeling showed that dung beetles can use reorientation to move away from the dung pile more efficiently. In this work, we study if reorientation can help beetles avoid competition (i.e., avoid having their dung balls captured), and if so, under what circumstances? This is investigated by implementing a model with two different type of beetles, a roller with a dung ball and a searcher which seeks to capture that dung ball. We show that reorientation can help rollers avoid searchers in a wide range of conditions, but that there are some circumstances in which rolling without reorienting can be a beetle's optimal strategy. We also show that rollers can minimize the probability that their dung ball is captured without making precise measurements of the time interval between dances or the angular deviation for dances.
\end{abstract}

Keywords Dung beetles · Competition · Random walk · Optimization

\section{Introduction}

For dung beetles, dung is a highly prized resource. Many dung beetles shape a portion of dung at a dung pile into a ball and roll it away, for burial and

University of California, Los Angeles

520 Portola Plaza, Los Angeles, CA 90095

Harvey Mudd College

320 E Foothill Blvd, Claremont, CA 91711

Tel.: (818)-399-7354

ORCID iD: 0000-0001-5445-9915

*Corresponding author. E-mail: lzinnbrooks@g.hmc.edu 
later consumption 1, 2]. These beetles are known as rollers. Some rollers, in order to save the labor of making their own dung balls, attempt to steal others' balls instead 2,8. Hence, competition for dung balls at or near the dung pile is very common. In the vicinity of large dung piles, as many as thousands of dung beetles and dozens of dung beetle species have been observed. Fights over a dung ball can occur between dung beetle species or within the same species 9. Naturally, rollers seek to avoid these fights as much as possible, since even rollers that win their fight (and keep their dung ball) may incur a significant energy cost. Hence, a roller's task is to move away from the dung pile as efficiently as possible 1,5]. Maintaining a straight line rolling path away from the pile is optimal for this purpose, but many factors make this path difficult to achieve. Namely, beetles are facing backwards while pushing dung balls larger than themselves, imperfections in the dung ball can make balls drift off course, and beetles need to roll their balls around obstacles and over uneven ground 1, 10].

Research has shown that dung beetles use celestial cues, including the sun, the moon, polarized light, and the Milky Way, to navigate [3 6]. Prior to rolling a dung ball away from the dung pile, most beetles perform a "dance" (a sequence of rotations and pauses) atop the ball. This dance allows beetles to get their bearings and to choose a rolling direction 1, 3. . Dances have also been observed later in the rolling process, particularly when beetles lose control of dung balls or are driven off their intended course by obstacles or uneven ground 1]. This shows that beetles use dances not only to select an initial rolling direction, but also to help maintain a straight line bearing (i.e., to reorient themselves).

Experiments by Baird et al. 1] have given us insight into how beetles choose the timing of dances. The authors constructed straight and semicircular tunnels, and directed rollers into these tunnels. Beetles danced significantly more often after emerging from a semicircular tunnel than after a straight tunnel [1]. This indicates that the dung beetle dance is a reorientation behavior. While beetles directed 180 degrees off course usually performed dances, the authors did not observe a consistent angular deviation (i.e., angle between the current path and a straight line path) at which rollers danced in the field [1]. So, perhaps beetles reorient themselves when there is a major disturbance in their path (i.e., a forced u-turn or losing control of a ball).

In order to further investigate the rolling and reorientation process, Yin and Zinn-Bjorkman 11 formulated a mathematical model of dung ball rolling paths in a circular domain. The authors modeled the rolling process using a persistent random walk, in which the rolling direction at each time step was drawn from a wrapped normal distribution centered at the previous rolling direction, the idea being that dung balls would tend to drift off their intended course over time. They also incorporated dancing (reorientation) by redirecting beetles to the "optimal direction" (direction of the shortest path to the boundary of the domain). In addition to being redirected, beetles incurred a "time penalty" from reorientation (i.e., they remained stationary for a given amount of time). The framing question from this work was "How often and 
under what circumstances should dung beetles reorient in order to minimize the average time to reach the boundary?"

Spending as little time as possible in the vicinity of the dung pile seems like an ideal way to reduce competition for dung balls. However, in this work, we study competition more directly by introducing a second type of beetle to the model from 11]. This beetle, which we call a "searcher," randomly moves in the domain, then moves directly towards the roller when the roller and searcher are sufficiently close (i.e., when the roller has been detected). This model allows us to address if reorientation can help rollers avoid fights, and if so, under what circumstances.

We begin the next section by recapping previous mathematical modeling in 11]. In Section 2.2, we describe our model for dung beetle competition, which we refer as the "roller-searcher model." In Section 3, we implement our rollersearcher model, and investigate how various parameters affect the percentage of beetles captured by searchers. We study this "percentage captured" in the presence and absence of reorientation (Section 3.1), in the presence of detection error (noise) in reorientation (Section 3.2), as detection radius of searchers varies (Section 3.3), and finally, when there are multiple searchers present (Section 3.4.

\section{Description of the roller-searcher model}

2.1 Movement of the rolling beetle: recap of previous mathematical modeling

Yin and Zinn-Bjorkman 11] developed a simple mathematical model to describe the movement of ball-rolling dung beetles. In this model, beetles were placed (with their dung balls) in the center of a circular arena of radius $R$ (i.e., at the origin $(x, y)=(0,0))$, and each beetle was given a random initial rolling direction. In the absence of "dances," movement of beetles was simulated using a persistent random walk, in which beetles would tend to "drift" away from their current rolling direction (due to the unevenness of the ground beneath them), with each new rolling direction drawn from a wrapped normal distribution with standard deviation $\sigma_{d}$, centered at the previous rolling direction (see [11], Algorithm 1). This parameter $\sigma_{d}$ was called the "drifting tendency" of the beetle. Each simulation ended when the beetle reached the boundary of the circular domain (i.e., when $x^{2}+y^{2} \geq R^{2}$ ).

The authors then incorporated dancing into this "basic drifting" model. Each time a dance was performed, the model beetle was reoriented to the direction of the shortest path to the boundary (designated as the "optimal direction"), while also being given a "time penalty," representing the duration of a dance. The authors considered two strategies for dancing: periodic reorientation, in which the time $T$ between consecutive dances was fixed, and error threshold reorientation, in which beetles danced only when their "error" $\theta_{e}$ (angle between optimal and current rolling directions) eclipsed a certain threshold (see [11, Algorithms 2 and 3). 
Yin and Zinn-Bjorkman 11] compared their simulation results with real data from dung beetles in model arenas 4], predicted the behavior of beetles in varying ground and celestial conditions, and suggested future experiments that would be illuminating. However, a limitation of the authors' model was that they did not address competition between beetles. Instead, each beetle was treated as independent (i.e., beetles were placed in the model domain one at a time), as in experiments by Dacke et al. [4]. In this work, we use the same mathematical models as 11 to simulate a rolling beetle (a "roller"), but we add an additional beetle, which we call a "searcher," whose aim is to find the roller and steal its dung ball. The goal of the roller is to reach the boundary of the circular domain before its dung ball is stolen (subsequently referred to as "captured") by the searcher. Hence, the roller's aim is to minimize the probability of its dung ball being captured. This is a different optimization problem to the one discussed in [11], in which the goal of the model beetle was to minimize its time taken to reach the boundary. In Table 1, we list the parameters and variables used for the rolling beetle.

\begin{tabular}{cl}
\hline Parameter/Variable & Interpretation \\
\hline$N$ & Number of dung beetles \\
\hline$v$ & Rolling speed (constant) \\
\hline$R$ & Radius of arena \\
\hline$L$ & Time needed to first reach the boundary \\
\hline$\Delta t$ & Length of rolling path to the boundary \\
\hline$\theta(t)$ & Time step \\
\hline$\sigma_{d}$ & Direction of rolling beetle at time $t$ \\
\hline$t_{\text {pen }}$ & Drifting tendency \\
\hline$T$ & Reorientation time penalty \\
\hline$\theta_{e}$ & Time interval between reorientations \\
\hline$\phi(t)$ & Error threshold for reorientation \\
\hline$n_{r}$ & Optimal direction to boundary at time $t$ \\
\hline & Number of reorientations per rolling beetle \\
\hline
\end{tabular}

Table 1: Model parameters or variables for the rolling beetle, with their definitions.

2.2 Movement of the searching beetle

As discussed above, movement of our rolling beetle is described by Algorithms 1-3 in [11. On the other hand, the searching beetle in our model performs an unbiased random walk, starting from a random location in the circular domain. Every second (i.e., every 10 time steps in numerical simulations), the searching beetle chooses a new search direction from a uniform distribution on $[-\pi, \pi)$. However, if the searcher detects the roller, which occurs when the searcher and roller are within a critical distance $D_{s}$, the searcher changes its behavior. Instead of performing an unbiased random walk, it now 
chooses its direction at each time step so as to "beeline" directly towards the roller. Searching beetles move at speed $v_{s}$ throughout simulations. We use reflecting boundary conditions for the searcher, so that the searcher never leaves the domain. The simulation ends when one of the following occurs:

- The searcher and roller are within a critical distance $R_{s}$ of one another. The roller's dung ball is considered "captured" in this case.

- The roller has moved outside of the domain (i.e., the stopping condition from [11]). This is called the "escaped" case.

For a table of parameters for the searching beetle, see Table 2 , and see the supplementary material for an algorithm of our roller-searcher model.

\begin{tabular}{cl}
\hline Parameter & Interpretation \\
\hline$D_{s}$ & Detection radius of the searcher \\
\hline$R_{s}$ & Capture radius of the searcher \\
\hline$v_{s}$ & Speed of the searcher \\
\hline$\sigma_{d t}$ & Noise level for periodic reorientation (see sec. 3.2 \\
\hline$\sigma_{d e}$ & Noise level for error threshold reorientation (see sec. 3.2 \\
\hline$n_{s}$ & Number of searchers (see sec. 3.4 \\
\hline
\end{tabular}

Table 2: Model parameters for the searching beetle, with their definitions.

In Fig. 1. we display sample paths for the roller and searcher for each of the scenarios described above. In Fig. 1(a), the searcher detects the roller near the boundary of the domain, beelines toward the roller, and captures its dung ball. In Fig. 1(b), the searcher also detects the roller near the boundary of the domain, but the roller escapes the domain prior to being captured. In this figure (and in all subsequent simulations), we use rolling speed $v=3.83 \mathrm{~cm} / \mathrm{s}$ and searching speed $v_{s}=5 \mathrm{~cm} / \mathrm{s}$. Default parameter values for $D_{s}$ and $R_{s}$ are $20 \mathrm{~cm}$ and $5 \mathrm{~cm}$, respectively $\left(D_{s}\right.$ will be varied in Section 3.3 .

\section{Results}

3.1 Choosing the optimal reorientation period and error threshold

In their previous study, Yin and Zinn-Bjorkman [11] showed that "dancing" (reorientation) improves mean first passage time to the boundary of the domain over "drifting" alone, over a wide range of drifting tendencies $\sigma_{d}$. Reorientation introduces a balancing act: frequent reorientations lead to a very straight path, but the beetle will spend a long time performing dances. The authors found that, as $\sigma_{d}$ (which represents unevenness of the ground) increases, it is optimal to increase the frequency of reorientations [11].

Our optimization problem is different, though related: our model rolling beetles seek to minimize the probability that they are captured by a searcher before exiting the domain. Intuitively, reducing the amount of time that rollers 


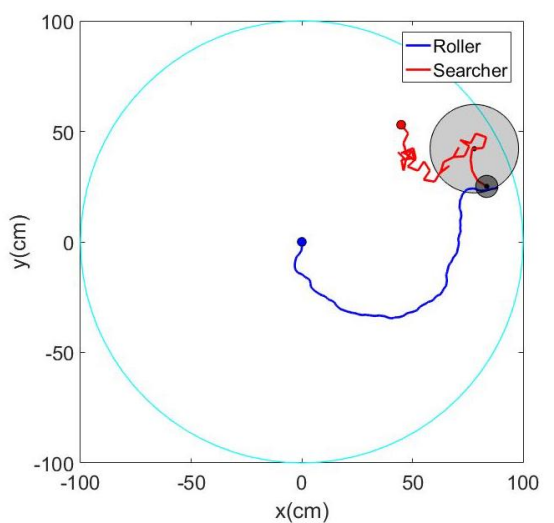

(a) Roller captured by searcher

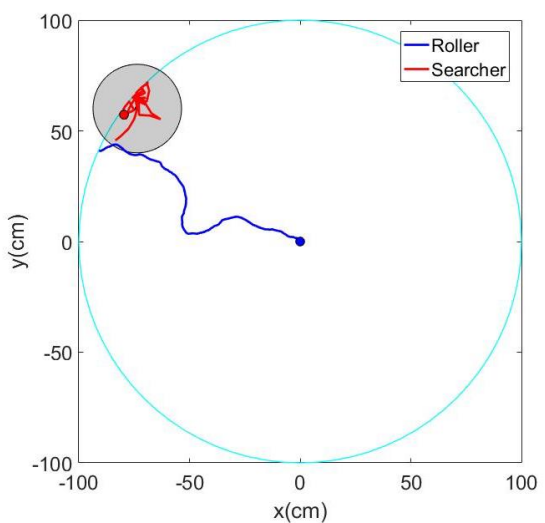

(b) Roller escapes from searcher

Fig. 1: Examples of capture and escape in the roller-searcher model. The blue curve shows the path of the rolling beetle, which performs a persistent random walk with drifting tendency $\sigma_{d}=\pi / 6$, while the red curve shows the path of the searcher. The starting positions of the roller and searcher are represented by blue and red dots, respectively. The light gray circle represents the detection radius for the searcher, while the dark gray circle (in Fig. (a) only) represents its capture radius.

spend in the domain would decrease their probability of being captured. However, rollers are stationary during reorientations, meaning that they will have no opportunity to escape beelining searchers if detected during a dance. Hence, it is not obvious that reorientation will be beneficial in this case.

In order to test the effect of reorientation on capture probability, we simulate 500 trials of our roller-searcher model for a wide range of drifting tendencies, from $\sigma_{d}=2 \pi / 60$ to $\sigma_{d}=31 \pi / 60$, with increments of $\pi / 60$. Then, for each value of $\sigma_{d}$, we calculate the percentage of trials that end with the rolling beetle being captured. We repeat these simulations for three different rolling strategies: no reorientation (the basic drifting model), periodic reorientation, and error threshold reorientation. For the latter two strategies, we find the optimal reorientation period $T^{*}$ and reorientation error threshold $\theta_{e}^{*}$ that minimize the probability of being captured. Results for percentage captured are shown in Fig. 2 (for full results, see supplementary Table S1).

We find that both reorientation techniques substantially decrease percentage captured from the no reorientation case. As drifting tendency $\sigma_{d}$ increases, reorientation becomes more and more beneficial. We also see that error threshold reorientation is the superior technique to periodic reorientation for $\sigma_{d}>\pi / 4$, but for lower drifting tendencies, there is very little difference in percentage captured. Finally, we see that for all three cases, percentage captured trends upward as $\sigma_{d}$ increases. This makes sense since rolling beetles take longer on average to reach the boundary of the domain as $\sigma_{d}$ increases 11 . 


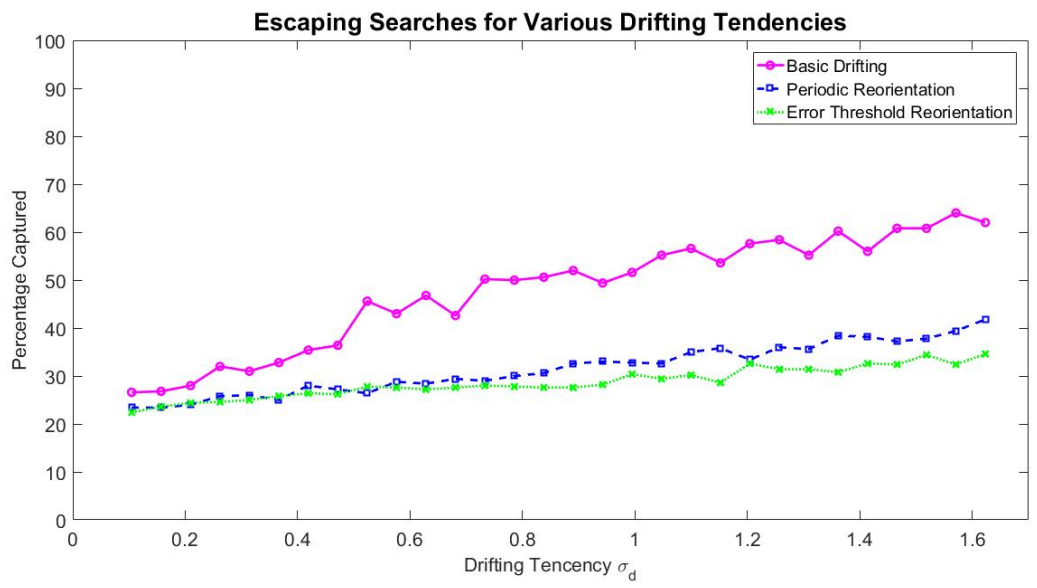

Fig. 2: Percentage of rolling beetles captured (out of 500 total) for three different rolling strategies: basic drifting (no reorientation), periodic reorientation with optimal period $T^{*}$, and error threshold reorientation with optimal error threshold $\theta_{e}^{*}$.

In Fig. 3. we display how the optimal reorientation period $T^{*}$ and optimal error threshold $\theta_{e}^{*}$ depend on drifting tendency $\sigma_{d}$. We find that $T^{*}$ generally decreases as $\sigma_{d}$ increases, while $\theta_{e}^{*}$ generally decreases (although there are several outliers, particularly for lower drifting tendencies). This indicates that, as the ground gets more uneven, beetles using periodic reorientation should reorient more frequently, while beetles using error threshold reorientation should tolerate larger deviations from the optimal path. These results are in agreement with those of [11 for mean first passage time.

How important is it for the rolling beetle to precisely choose the optimal reorientation strategy? To address this question, we choose a single drifting tendency $\sigma_{d}=\pi / 4$ and compute the percentage of beetles captured (out of 500 total) for a broad range of reorientation periods and error thresholds. We find that, for both reorientation strategies, there is a wide range of choices that produces a capture percentage close to the minimum (see Fig. 4). For example, any choice of reorientation period between $T=1.8 \mathrm{~s}$ and $T=9 \mathrm{~s}$ or error threshold between $\theta_{e}=38 \pi / 90$ and $\theta_{e}=76 \pi / 90$ gives a percentage captured within 5 percentage points of the optimum.

\subsection{Effect of detection error in reorientation}

In the previous section, we showed that a broad range of choices for reorientation periods or error thresholds yield similar success in avoiding searchers. This suggests that rolling beetles do not need to be extremely precise in the timing of reorientations in order to achieve close to optimal results. In this sec- 


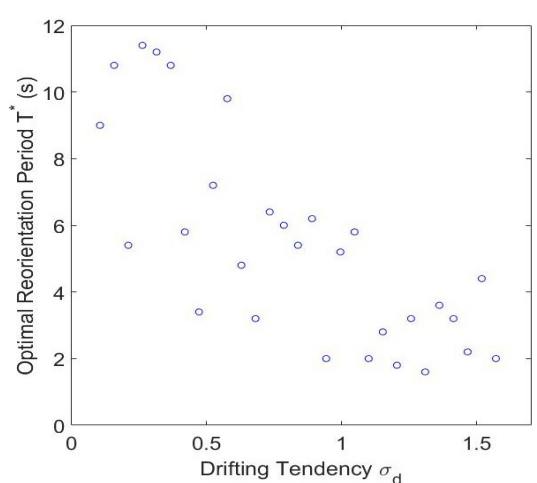

(a)

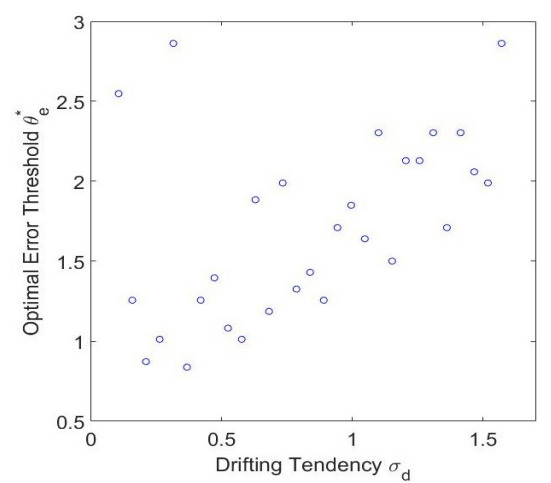

(b)

Fig. 3: Reorientation period (Fig. a) and error threshold (Fig. b) that minimize percentage of beetles captured, for a broad range of drifting tendencies $\left(\sigma_{d}=\right.$ $2 \pi / 60,3 \pi / 60, \ldots, 31 \pi / 60)$.

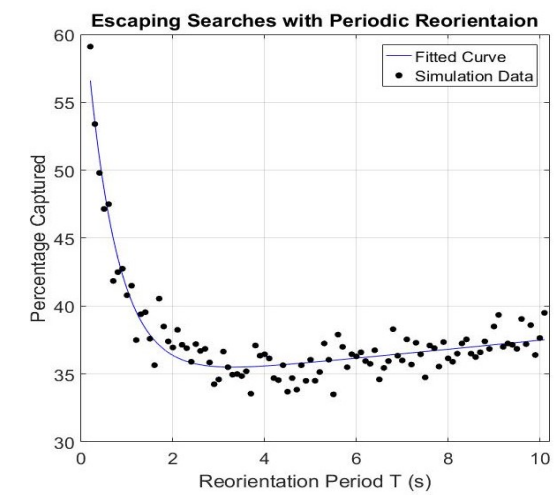

(a)

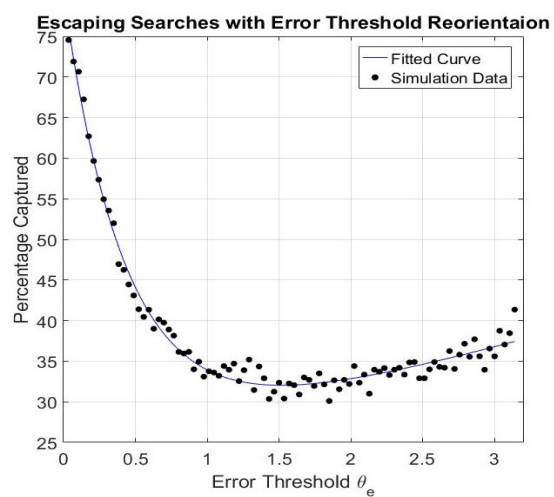

(b)

Fig. 4: Percentage of beetles captured for various reorientation periods (Fig. a) and error thresholds (Fig. b), together with best-fit curves.

tion, we explore this idea further by adding a noise component (i.e., a detection error) to periodic and error threshold reorientation.

For "noisy" periodic reorientation, rolling beetles choose a reorientation period $T$, but cannot measure this time precisely. Instead, the time $T_{a}$ between successive reorientations is chosen as follows:

$$
T_{a}=T+Z, \quad Z \sim N\left(0, \sigma_{d t}\right) .
$$

Here, the random variable $Z$ is sampled from a normal distribution with mean 0 and standard deviation $\sigma_{d t}$. We refer to $\sigma_{d t}$ as the detection error for peri- 
odic reorientation. Similarly, for "noisy" error threshold reorientation, rolling beetles cannot precisely choose the same error threshold $\theta_{e}$ each time they reorient. Instead, the error threshold $\theta_{a}$ between each reorientation is

$$
\theta_{a}=\theta_{e}+Z, \quad Z \sim N\left(0, \sigma_{d e}\right)
$$

where $\sigma_{d e}$ is called the detection error for error threshold reorientation.

To test whether noise has a detrimental effect on capture probability, we begin by choosing a single drifting tendency $\sigma_{d}=\pi / 4$. We then implement ten different levels of detection error (noise): $\sigma_{d t}=0.1,0.2, \ldots, 1 s$ for periodic reorientation and $\sigma_{d e}=\pi / 90,2 \pi / 90, \ldots, 10 \pi / 90$ for error threshold reorientation. For each noise level, we find the optimal reorientation period $T^{*}$ and error threshold $\theta_{e}^{*}$, then find the percentage of rolling beetles captured (out of 500 total) for these optimal values. We then compare these capture percentages to those for the zero noise case. Results are shown in Fig. 5. We find that noise does not have a significant effect on percentage captured for $\sigma_{d}=\pi / 4$.

Does noise have more influence for other drifting tendencies? To address this question, we choose a single value for detection error $\left(\sigma_{d t}=1 \mathrm{~s}\right.$ for periodic reorientation, $\sigma_{d e}=10 \pi / 90$ for error threshold reorientation), and find the optimal percentage captured for values of $\sigma_{d}$ from $2 \pi / 60$ to $31 \pi / 60$, with increments of $\pi / 60$. We then compare these capture percentages to those from the no-noise case (see Fig. 2, blue and green curves). Results are shown in Fig. 6. We find that, again, noise has no discernible effect on percentage captured. The results from this section reinforce that our model beetles do not need to make precise measurements in order to achieve close to optimal results.

\subsection{Varying detection ability of searchers}

In the previous two sections, we used detection radius $D_{s}=20 \mathrm{~cm}$ for searchers. Ground conditions could potentially influence this radius. For example, uneven ground with obstacles could obscure searchers' vision, while even, unobstructed ground could allow for detection of rollers over a longer distance. In this section, we simulate our model with drifting tendency $\sigma_{d}=\pi / 4$, while varying $D_{s}$ from $5 \mathrm{~cm}$ to $50 \mathrm{~cm}$, with increments of $5 \mathrm{~cm} . D_{s}=5 \mathrm{~cm}$ indicates no detection ability for searchers, since $D_{s}$ equals the capture radius $R_{s}$ in this case. For each value of $D_{s}$, we run 500 trials for each rolling strategy (basic drifting, as well as optimal periodic and error threshold reorientation).

Results for percentage captured are shown in Fig. 7. We find that percentage captured increases roughly linearly with $D_{s}$. Reorientation provides a fairly consistent amount of benefit over the drifting model over the range of values for $D_{s}$, with error threshold reorientation remaining the slightly superior method to error threshold reorientation. Full results, including optimal values $T^{*}$ and $\theta_{e}^{*}$ for each value of $D_{s}$, are shown in supplementary Table S2. 
(a)

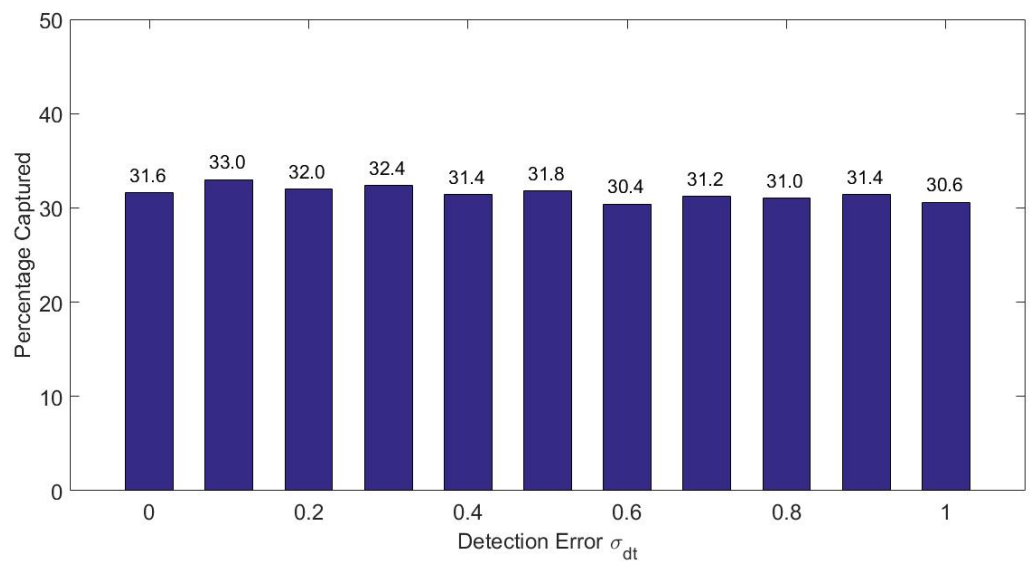

(b)

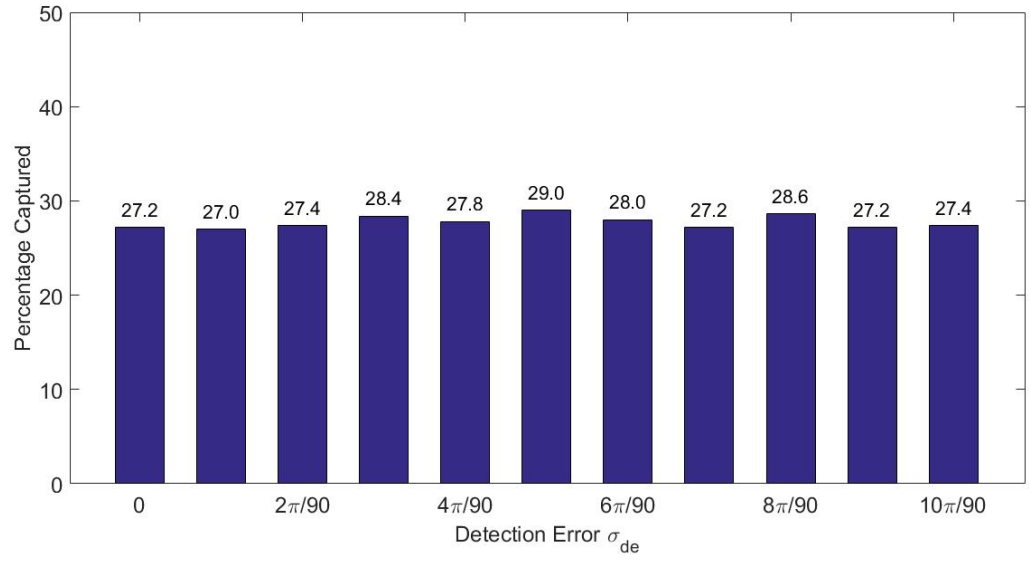

Fig. 5: Percentage of beetles captured for various levels of detection error, for periodic reorientation (Fig. a) and error threshold reorientation (Fig. b).

\subsection{Escaping multiple searchers}

In the wild, of course, each roller is not necessarily pursued by a single searcher - it is very possible that a roller will need to evade several searchers during its journey with a dung ball [8,9]. In this section, we adjust our rollersearcher model to incorporate multiple searchers. Each searcher starts from a random location in the domain, and has the same movement behavior (unbiased random walk with beelining) as in the single searcher model (see Section 2.2. . Our simulations end when the roller escapes or when it is captured by one of the searchers. 
(a)

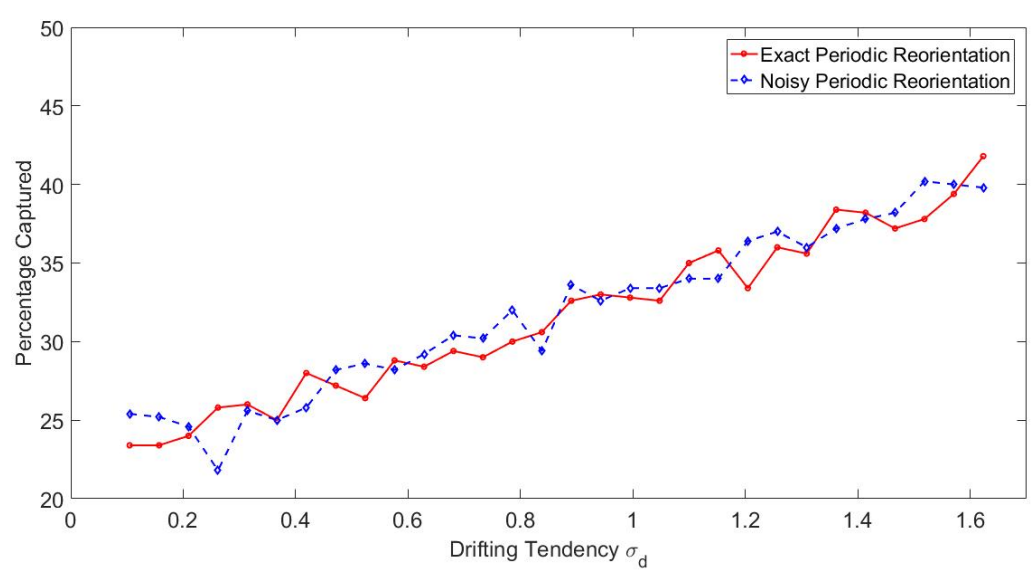

(b)

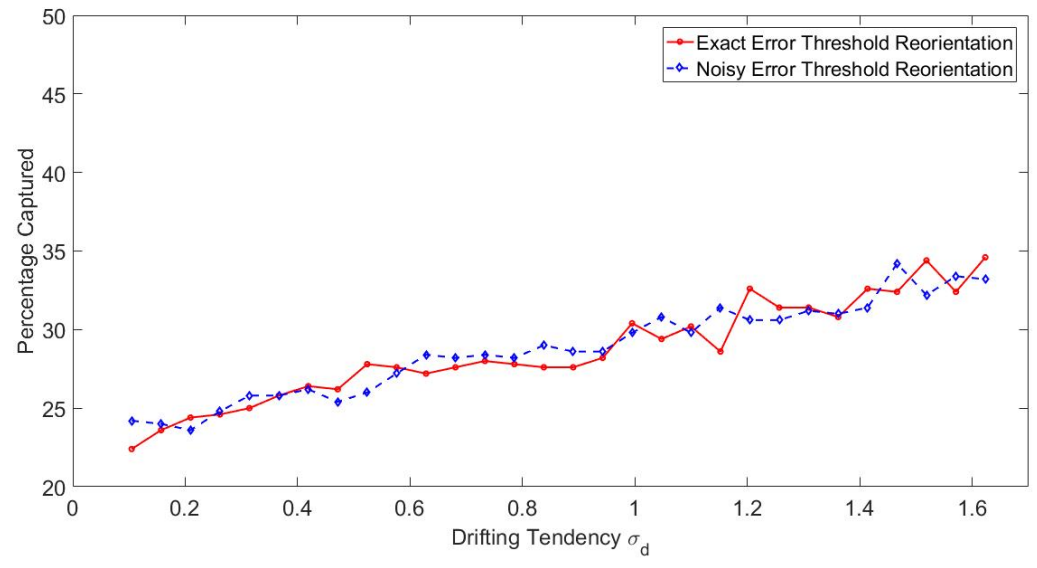

Fig. 6: Percentage of beetles captured for various drifting tendencies $\sigma_{d}$, for periodic reorientation (Fig. a) and error threshold reorientation (Fig. b). Red curves represent results for no noise $\left(\sigma_{d t}=0\right.$ and $\left.\sigma_{d e}=0\right)$, while blue curves represent results with noise implemented $\left(\sigma_{d t}=1 s\right.$ and $\left.\sigma_{d e}=10 \pi / 90\right)$.

We now simulate our roller-searcher model for number of searchers $n_{s}=$ $1,2,3, \ldots, 10,12$, and 15 , with drifting tendency $\sigma_{d}=\pi / 4$ and detection radius $D_{s}=20 \mathrm{~cm}$. For each value of $n_{s}$, we compute the percentage of rolling beetles captured (out of 500) for the basic drifting model, optimal periodic reorientation, and optimal error threshold reorientation. Results are shown in Fig. 8. Interestingly, we find that reorienting to escape searchers is no longer beneficial for $n_{s} \geq 4$. We also see that, as usual, error threshold reorientation is the slightly superior technique to periodic reorientation. We find that there 


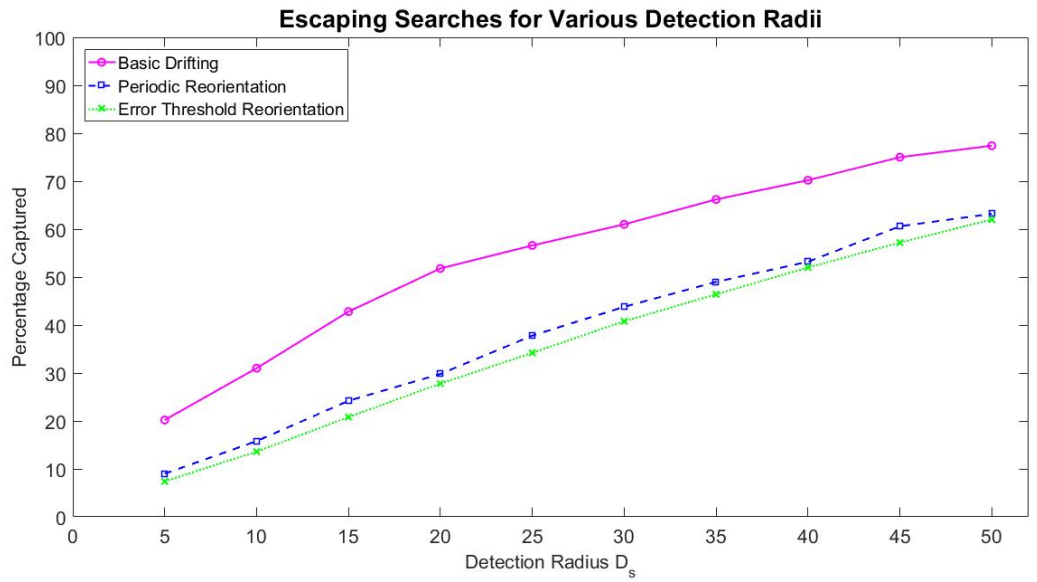

Fig. 7: Percentage of rolling beetles captured (out of 500 total) for the basic drifting model, periodic reorientation with optimal period $T^{*}$, and error threshold reorientation with optimal error threshold $\theta_{e}^{*}$, for a range of detection radii $D_{s}$ for searchers.

is no discernible trend for optimal period and error threshold as number of searchers increases (see supplementary Table S3).

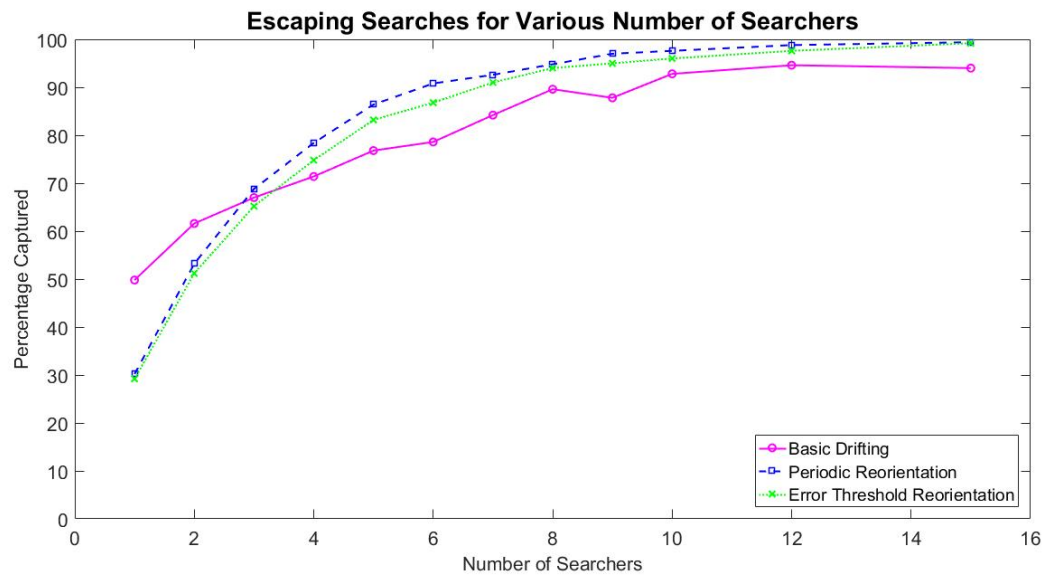

Fig. 8: Percentage of rolling beetles captured (out of 500 total) for three different rolling strategies and for various number of searchers. 


\section{Discussion}

In this work, we formulated and simulated a simple competition model for ball-rolling dung beetles. The competition occurs between a "roller," which has formed a dung ball and seeks to roll it away from the main dung pile, and a "searcher," which aims to discover the roller and steal its dung ball to save the labor of creating one itself. Although we do not include the battle for the dung ball (instead assuming that the roller's ball is "captured" as soon as it comes into close proximity with the searcher), as far as we are aware, this is the first mathematical model to address competition for dung balls.

Previous work by Yin and Zinn-Bjorkman [11] was motivated by experiments from Baird et al. [1] and Dacke et al. [4], who studied the behavior of isolated ball-rolling beetles. Hence, the mathematical model formulated in [11] focused on a single roller, in an effort to reasonably simulate ball-rolling paths. Many of the findings from 11] focused on the role of "dancing" (reorientation) in reducing time to reach the boundary of a circular domain. We were interested in the benefits that reorientation can provide rollers in avoiding battles for their dung ball.

In our model (as in [11]), reorientation is implemented either at fixed time intervals (for periodic reorientation) or at a specific angular deviation (for error threshold reorientation). Both methods would require unreasonably precise measurements from rollers in order to minimize the probability of being captured by searchers. However, we found that a broad range of values for $T$ (time period between reorientations) and $\theta_{e}$ (angular deviation from the optimal path) produce close to optimum results, and that reorientation with noise is equally effective to "perfect" reorientation. These are significant findings, as they indicate that reorientation can be an effective strategy for avoiding searchers even when timing of reorientations is unpredictable. Indeed, results from 11 have demonstrated that there is not a fixed angular deviation from a straight path at which rolling beetles dance. It would be interesting to also record the timing of dung beetle dances in the field, to test whether rollers might reorient periodically, and if they do, how well they can regulate the time between consecutive reorientations.

As there are often hundreds or even thousands of beetles at a dung pile, it is unreasonable to assume that each roller is pursued by a single searcher [9]. We therefore devoted Section 3.4 to study how adding additional searchers might change a roller's optimal strategy. Interestingly, we found that, for four or more searchers, reorientation is no longer a beneficial strategy (i.e., it is optimal to roll the dung ball without any course correction). This is likely because, as number of searchers increases, the time penalty associated with reorientation becomes more and more costly. Each time a roller remains stationary in our model, it gives searchers more opportunity to "beeline" towards it and capture its dung ball. Hence, rolling "blindly" (without reorientation) becomes an optimal method, as beetles using this strategy are always moving. This result could potentially be tested experimentally, either in the field or in a model 
arena, by observing whether or not a roller's behavior changes as its number of competitors increases.

A limitation of our model is that it does not directly account for obstacles in the way of the roller or the searcher. Incorporating obstacles into the rollersearcher model (by using a jump-diffusion model or similar) could be an interesting direction to pursue. Obstacles in the rolling path would seemingly be of greater detriment to rollers than to searchers, as rollers are facing backwards with a large dung ball blocking its view of the path ahead, while searchers are unencumbered and could potentially fly over obstacles 10. Movement of our searching beetle (random walk with beelining) is also very basic. If searchers' positions could be tracked in the field (as [4] did for rollers), we could perhaps formulate a more accurate model to describe movement of searchers.

Another limitation of our model is that we used a single fixed rolling speed and a single fixed searching speed. Rolling speed depends on the size and species of the beetle, the size of its dung ball, and terrain 10. Temperature has been shown to affect the movement of searching beetles - body temperature and the temperature of a beetles' environment pose limitations to its flying height and activity period [2,7]. It could be interesting to explore how the relative speed of roller and searcher dictates the roller's optimal strategy for avoiding competition. We've shown that rollers should change strategy depending on local terrain. However, this analysis could indicate whether or not rollers should change their strategy depending on the ambient temperature.

In future, we hope to investigate dung beetle competition more directly by including both the pursuit of dung balls and the fight over dung balls in our model. One potential approach could be to use an agent-based model, in which the probability of a beetle winning a "battle" for a dung ball depends on a variety of factors, including size, body temperature, as well the number and recency of prior battles. We could then use our model to answer interesting questions such as "How does the size of a roller affect the probability that it escapes with its dung ball?" and "How many times do we expect a dung ball to change hands before it is buried?"

\section{Declarations}

Funding: The authors did not receive support from any organization for the submitted work.

Conflicts of interest: The authors have no relevant financial or non-financial interests to disclose.

Ethics approval: Not applicable.

Consent to participate: Not applicable.

Consent for publication: Not applicable. 
Availability of data and material: Additional data provided in Supplementary Material.

Code availability: MATLAB scripts available on Github at https://github. com/JerryYinUCLA/Dung_Beetle_Competition

Author contributions: ZY wrote code, ran simulations, and composed figures and tables. LZ conceived of the study and helped to draft the manuscript. All authors read and approved the final manuscript.

\section{References}

1. Baird, E., Byrne, M.J., Smolka, J., Warrant, E.J., Dacke, M.: The dung beetle dance: an orientation behaviour? PLoS One 7(1), e30211 (2012)

2. Bartholomew, G.A., Heinrich, B.: Endothermy in african dung beetles during flight, ball making, and ball rolling. Journal of Experimental Biology 73(1), 65-83 (1978)

3. Byrne, M., Dacke, M., Nordström, P., Scholtz, C., Warrant, E.: Visual cues used by ball-rolling dung beetles for orientation. Journal of Comparative Physiology A 189(6), 411-418 (2003)

4. Dacke, M., Baird, E., Byrne, M., Scholtz, C.H., Warrant, E.J.: Dung beetles use the milky way for orientation. Current Biology 23(4), 298-300 (2013)

5. Dacke, M., Byrne, M., Smolka, J., Warrant, E., Baird, E.: Dung beetles ignore landmarks for straight-line orientation. Journal of Comparative Physiology A 199(1), 17-23 (2013)

6. Dacke, M., Nordström, P., Scholtz, C.H.: Twilight orientation to polarised light in the crepuscular dung beetle scarabaeus zambesianus. Journal of experimental biology 206(9), 1535-1543 (2003)

7. Gómez, V.C.G., Verdú, J.R., Zurita, G.A.: Thermal niche helps to explain the ability of dung beetles to exploit disturbed habitats. Scientific reports 10(1), 1-14 (2020)

8. Hanski, I., Cambefort, Y.: Competition in dung beetles. Dung beetle ecology pp. 305$329(1991)$

9. Heinrich, B., Bartholomew, G.A.: Roles of endothermy and size in inter-and intraspecific competition for elephant dung in an african dung beetle, scarabaeus laevistriatus. Physiological Zoology 52(4), 484-496 (1979)

10. Tomkins, J.L., Simmons, L.W., Knell, R.J., Norris, K.A.: Correlates of ball size and rolling speed in the dung beetle kheper nigroaeneus (coleoptera: Scarabaeidae). Journal of Zoology 248(4), 483-487 (1999)

11. Yin, Z., Zinn-Björkman, L.: Simulating rolling paths and reorientation behavior of ballrolling dung beetles. Journal of Theoretical Biology 486, 110106 (2020) 


\section{Figures}

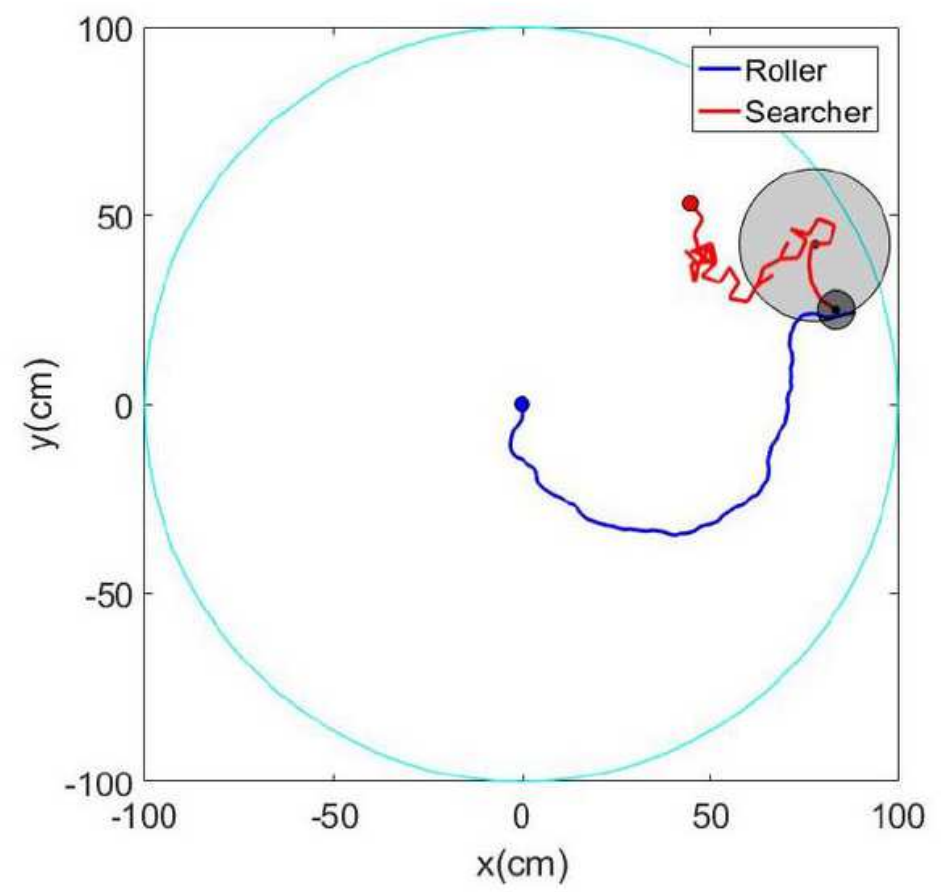

(a) Roller captured by searcher

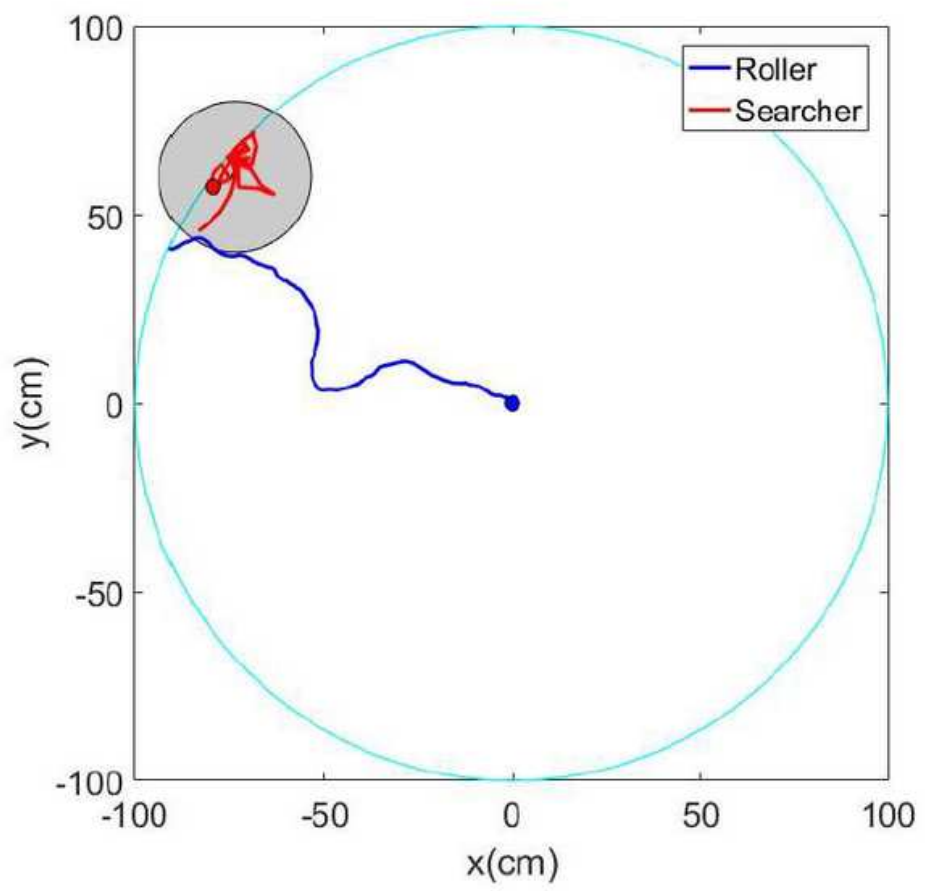

(b) Roller escapes from searcher

\section{Figure 1}

Examples of capture and escape in the roller-searcher model. The blue curve shows the path of the rolling beetle, which performs a persistent random walk with drifting tendency $\sigma d=\pi / 6$, while the red curve shows the path of the searcher. The starting positions of the roller and searcher are represented by blue and red dots, respectively. The light gray circle represents the detection radius for the searcher, while the dark gray circle (in Fig. (a) only) represents its capture radius. 


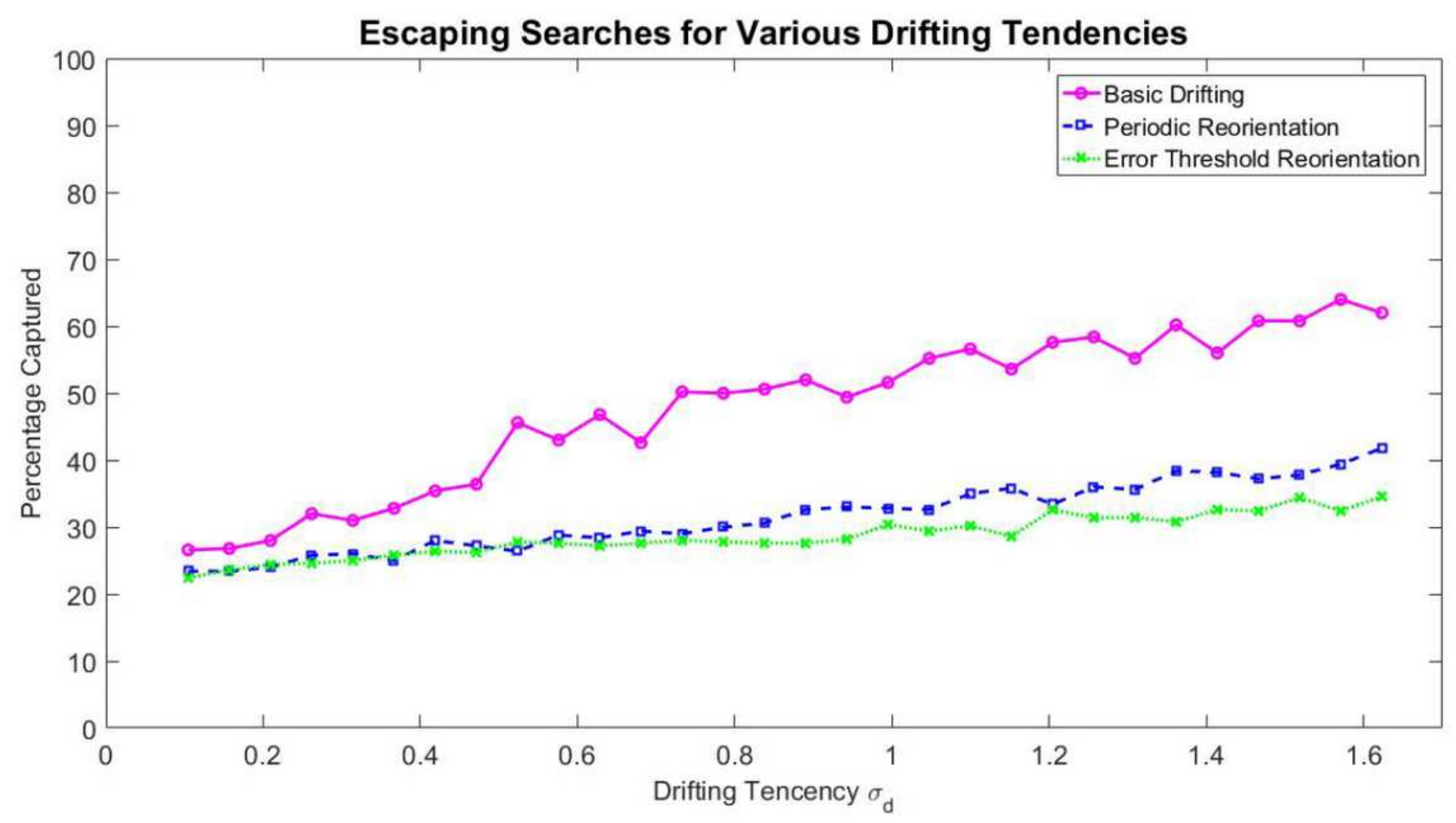

Figure 2

Percentage of rolling beetles captured (out of 500 total) for three differ-ent rolling strategies: basic drifting (no reorientation), periodic reorientation with optimal period $\mathrm{T} \otimes$, and error threshold reorientation with optimal error threshold $\theta$ e囚.

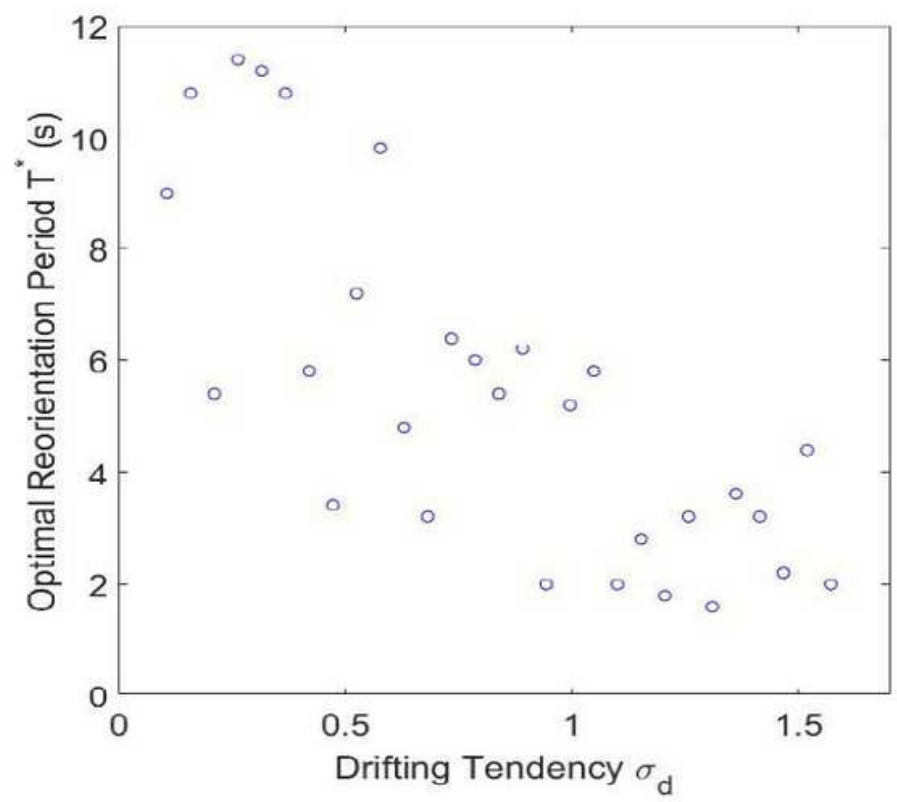

(a)

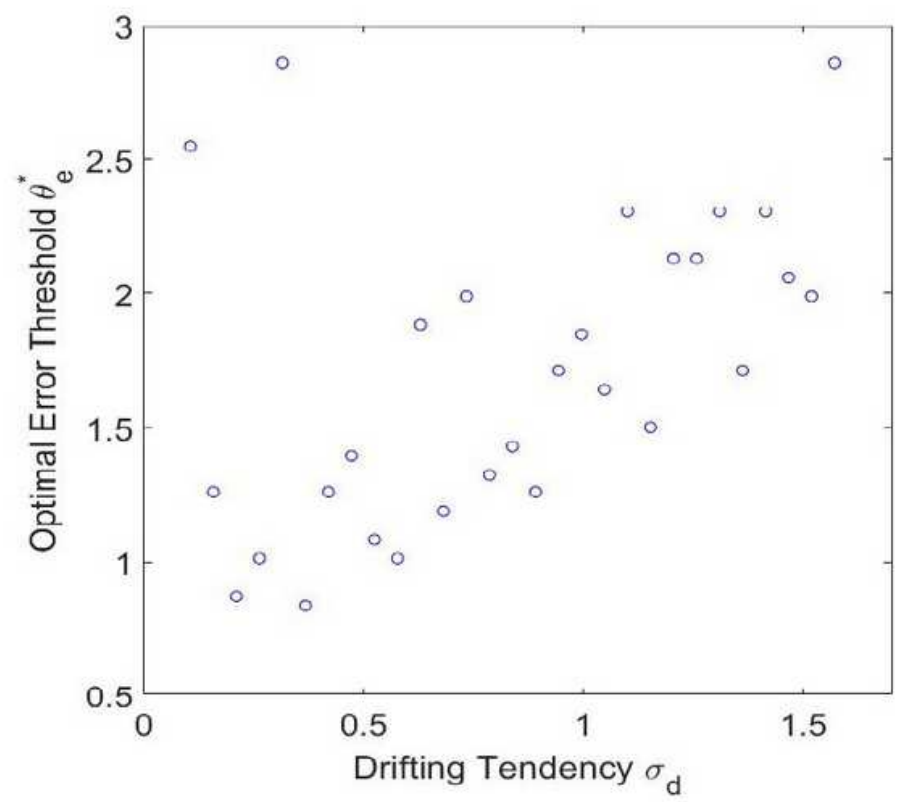

(b)

Figure 3 
Reorientation period (Fig. a) and error threshold (Fig. b) that minimize percentage of beetles captured, for a broad range of drifting tendencies $(\sigma d=2 \pi / 60,3 \pi / 60, \ldots, 31 \pi / 60)$.

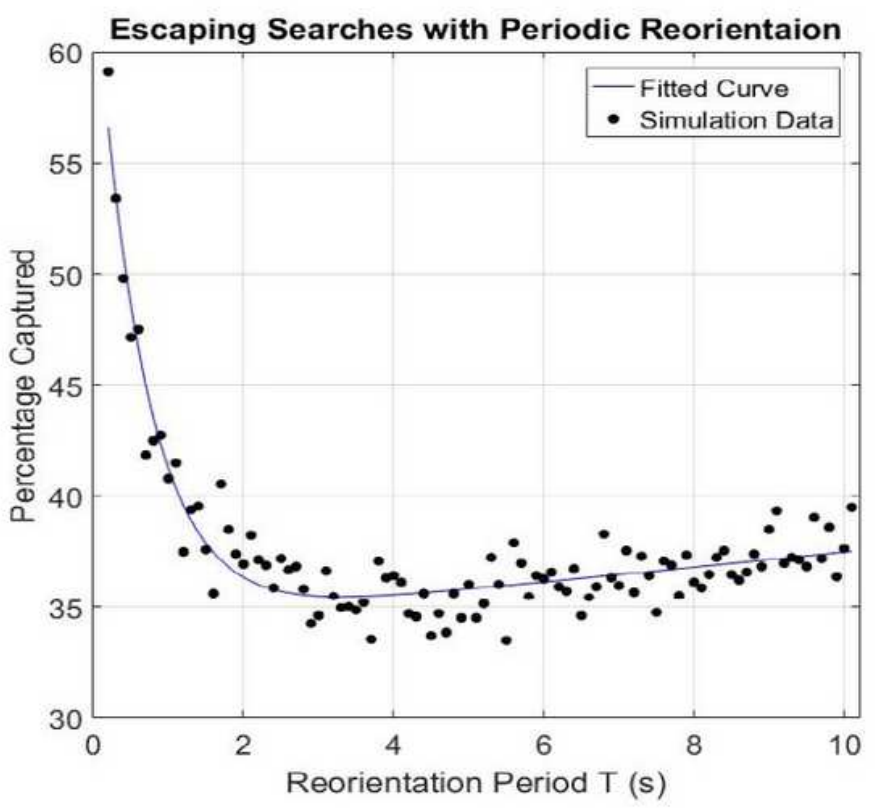

(a)

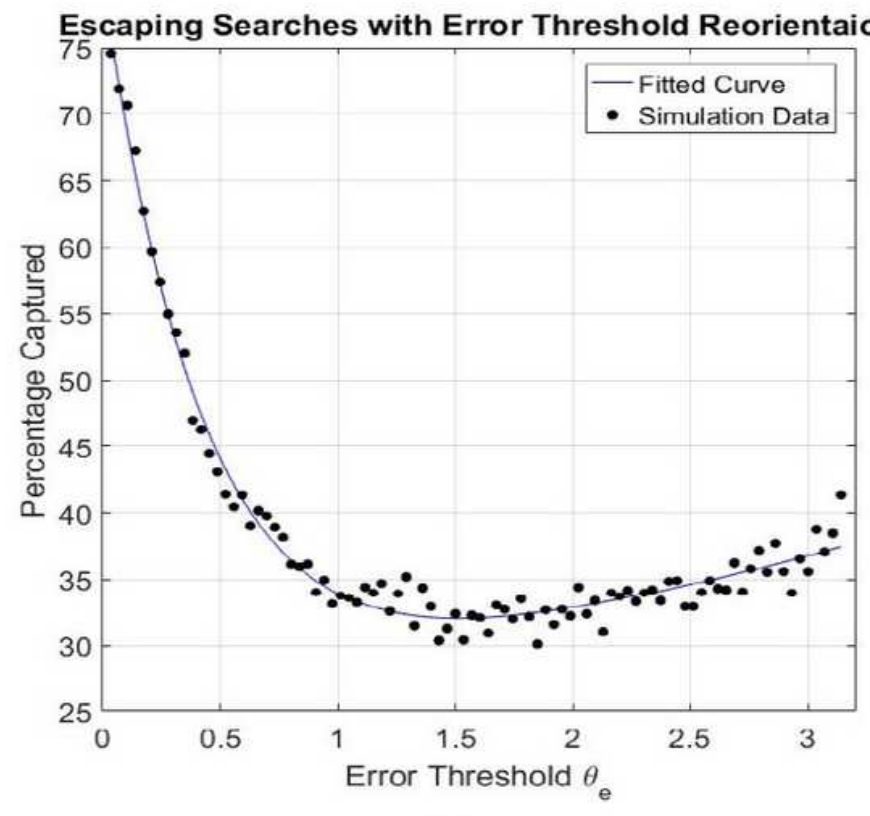

(b)

\section{Figure 4}

Percentage of beetles captured for various reorientation periods (Fig. a) and error thresholds (Fig. b), together with best-fit curves. 
(a)

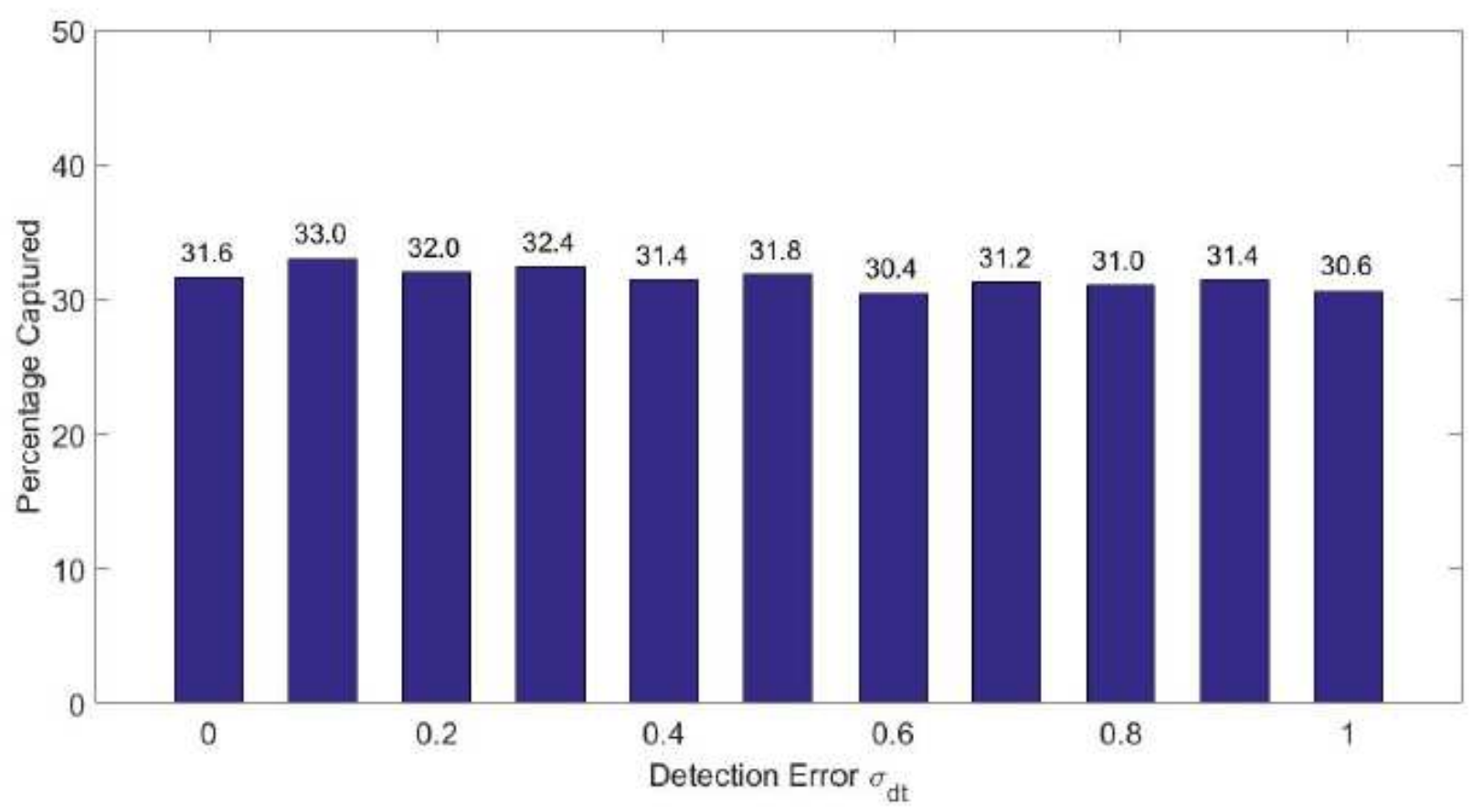

(b)

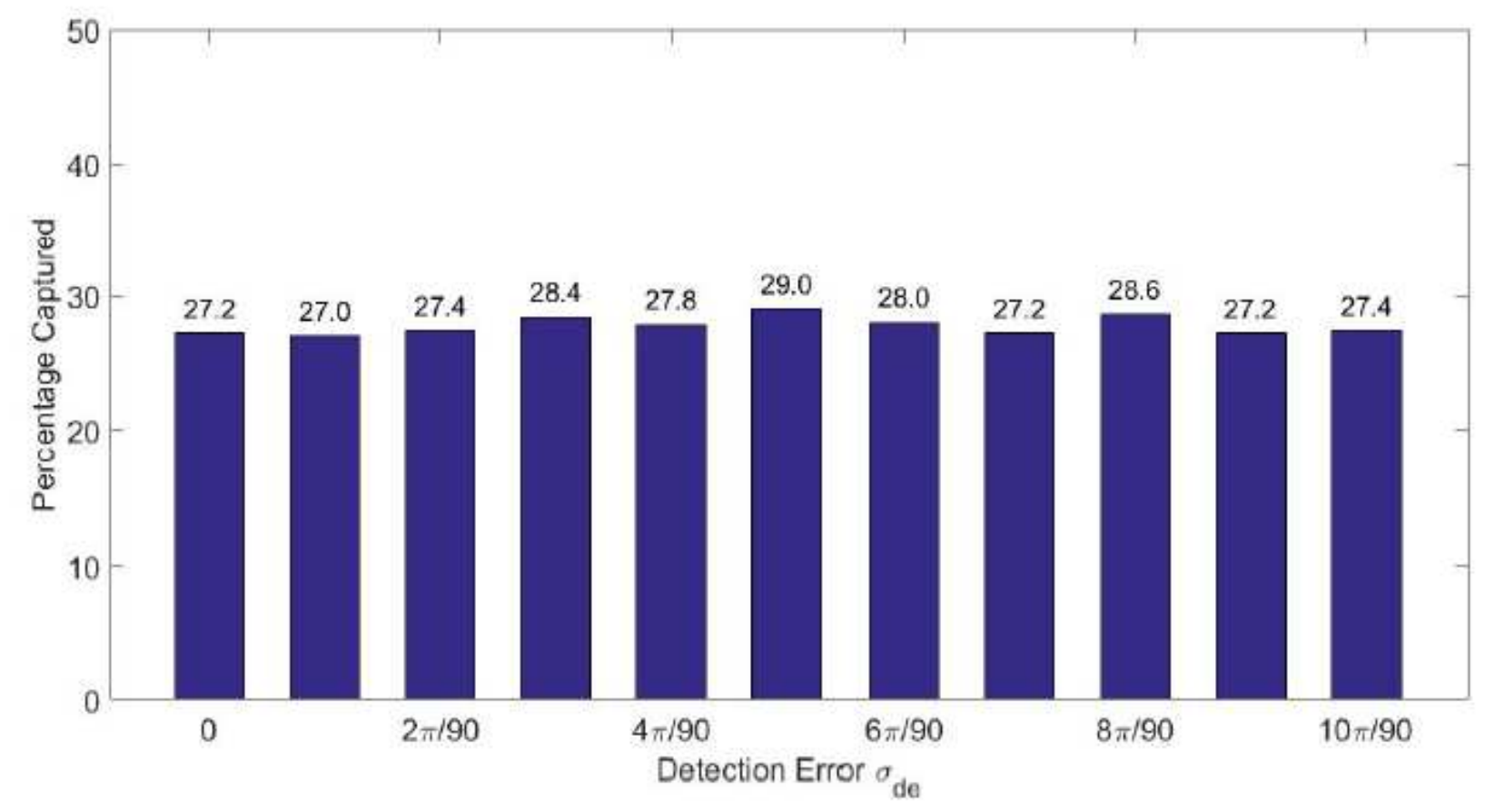

Figure 5

Percentage of beetles captured for various levels of detection error, for periodic reorientation (Fig. a) and error threshold reorientation (Fig. b). 
(a)

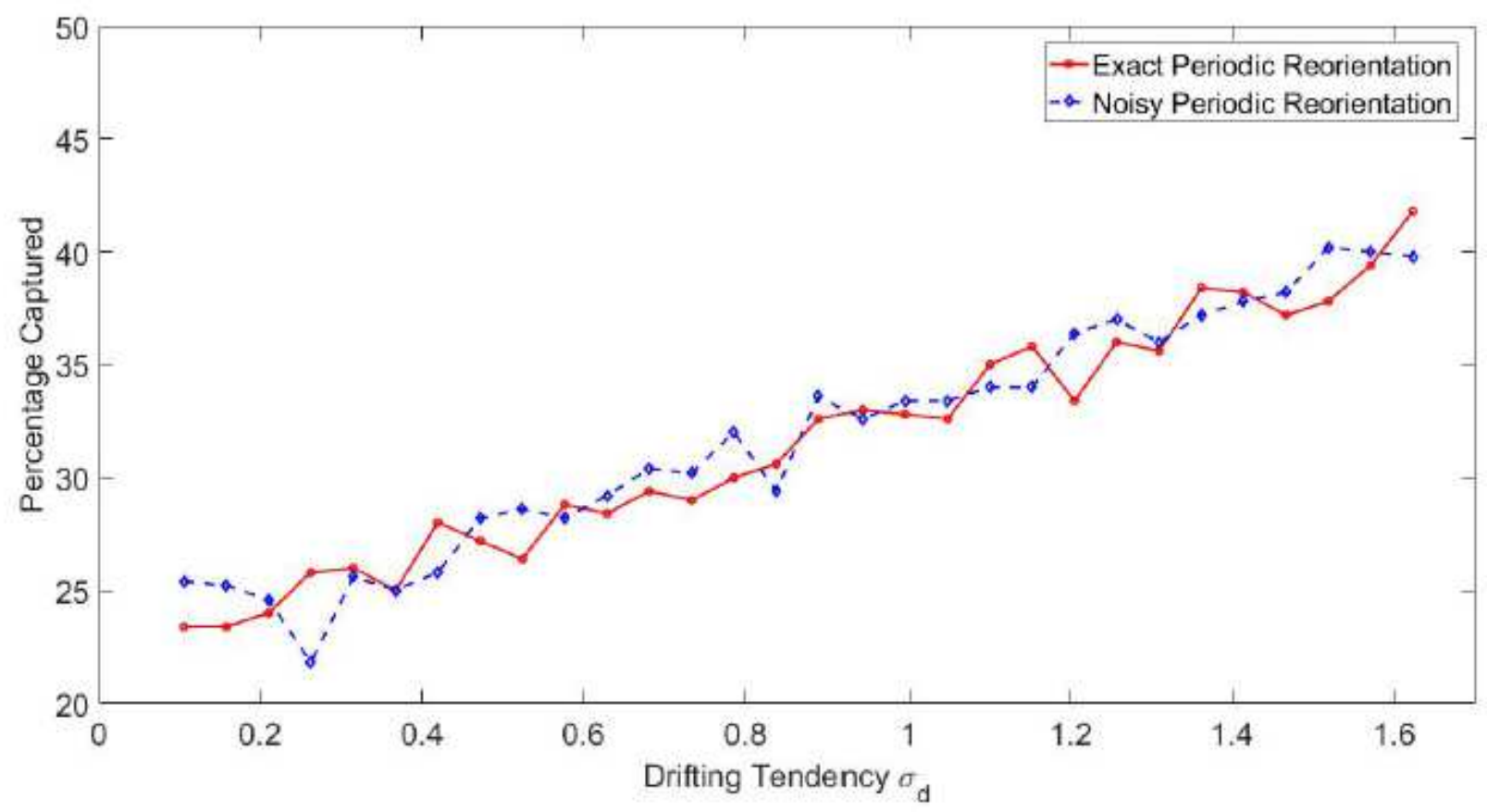

(b)

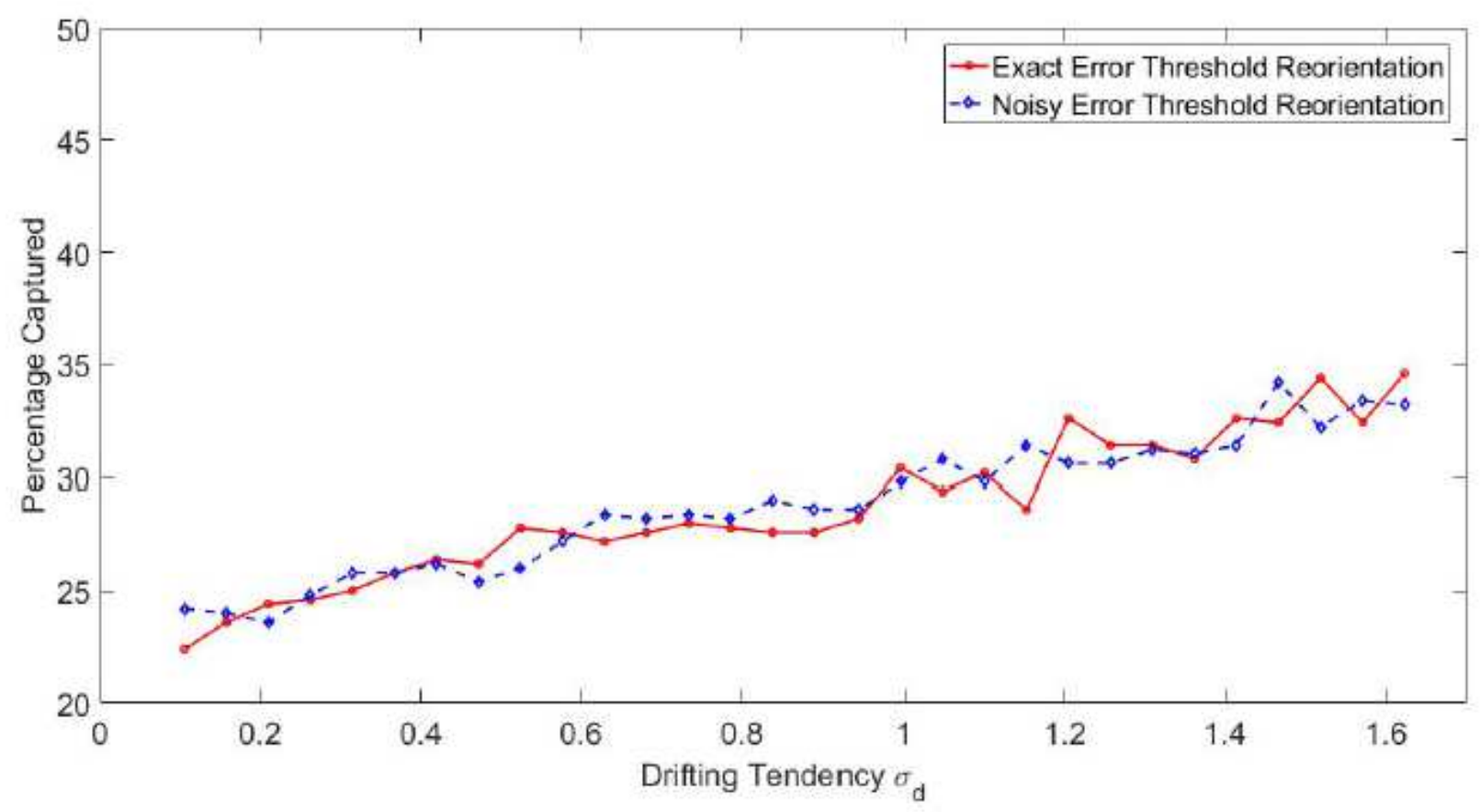

\section{Figure 6}

Percentage of beetles captured for various drifting tendencies od, for periodic reorientation (Fig. a) and error threshold reorientation (Fig. b). Red curves represent results for no noise ( $\sigma d t=0$ and $\sigma d e=0$ ), while blue curves represent results with noise implemented ( $\sigma \mathrm{dt}=1 \mathrm{~s}$ and $\sigma \mathrm{de}=10 \pi / 90)$. 


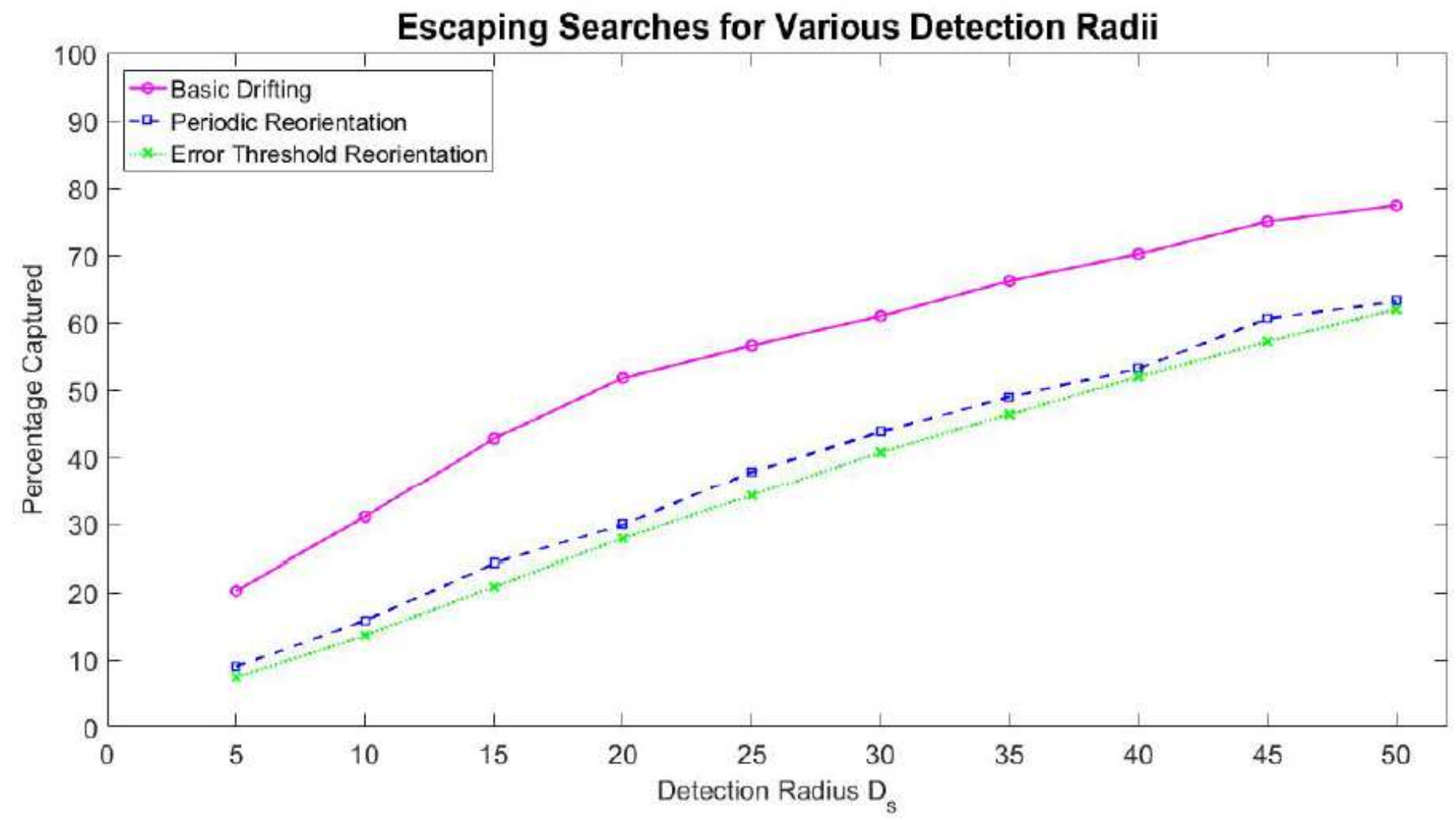

Figure 7

Percentage of rolling beetles captured (out of 500 total) for the basic drifting model, periodic reorientation with optimal period $\mathrm{T} \otimes$, and error threshold reorientation with optimal error threshold $\theta \mathrm{e} \bigotimes$, for a range of detection radii Ds for searchers. 


\section{Escaping Searches for Various Number of Searchers}

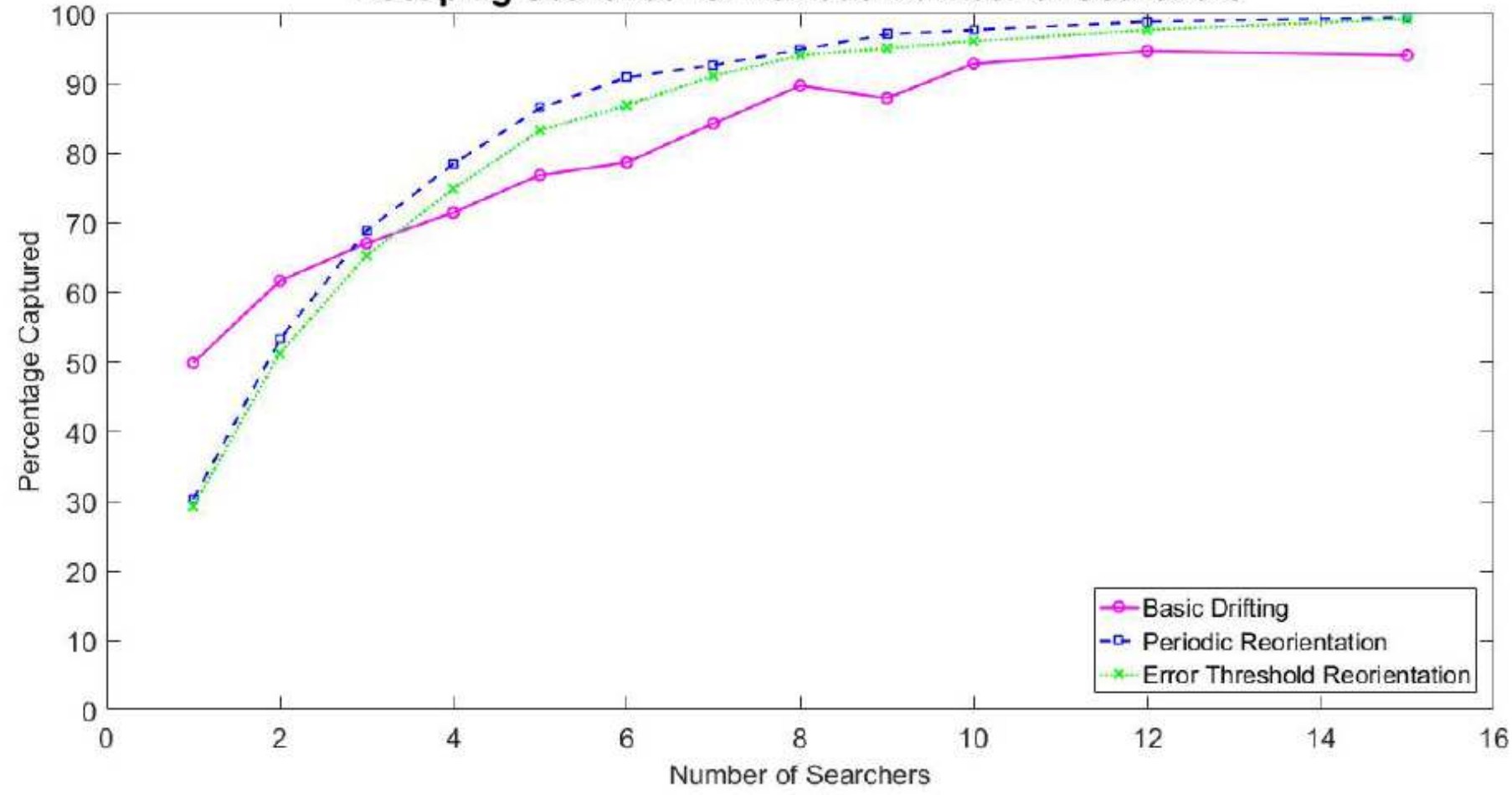

Figure 8

Percentage of rolling beetles captured (out of 500 total) for three different rolling strategies and for various number of searchers.

\section{Supplementary Files}

This is a list of supplementary files associated with this preprint. Click to download.

- SuppMaterial.pdf 